\title{
The role of hidden ambiguities in the linear sigma model with fermions
}

\author{
Brigitte Hiller $^{\mathrm{a}}$, A.L. Mota ${ }^{\mathrm{b}, \mathrm{e}, *}$, M.C. Nemes ${ }^{\mathrm{a}, \mathrm{c}}$, Alexander A. Osipov ${ }^{\mathrm{a}, \mathrm{d}}$, \\ Marcos Sampaio ${ }^{c}$ \\ a Centro de Física Teórica, Departamento de Física da Universidade de Coimbra, 3004-516 Coimbra, Portugal \\ b Departamento de Ciências Naturais, Universidade Federal de São João del Rei, São João del Rei, MG, Brazil \\ ${ }^{\mathrm{c}}$ Departamento de Física, Instituto de Ciências Exactas, Universidade Federal de Minas Gerais, \\ BH, CEP 30161-970, MG, Brazil \\ d Joint Institute for Nuclear Research, Laboratory of Nuclear Problems, 141980 Dubna, Moscow Region, Russia \\ e Departamento de Fisica Moderna, Faculdad de Ciencias, Universidad de Granada, Granada, Spain
}

Received 4 April 2005; received in revised form 30 January 2006; accepted 9 February 2006

Available online 21 February 2006

\begin{abstract}
The $U_{L}(3) \times U_{R}(3)$ linear sigma model (LSM) with quark degrees of freedom is used to show that radiative corrections generate undetermined finite contributions. Their origin is related to surface terms which are differences between divergent integrals with the same degree of divergence. The technique used to detect these ambiguities is an implicit regularization on basic divergent integrals that do not depend on external momenta. We show that such contributions are absorbed by renormalization or fixed by symmetry requirements. The general expression for surface terms is derived. Renormalization group coefficients are calculated, as well as relevant observables for this model, such as $f_{\pi}, f_{\kappa}$ and the pion and kaon form factors. (c) 2006 Elsevier B.V. All rights reserved.
\end{abstract}

\section{Introduction}

In dealing with ultraviolet infinities which stem from the semi-classical expansion in a quantum field theory, the renormalization program plays an essential role to fit the experimental data which the model is meant to describe. In regularizing and subtracting a divergence there appears

\footnotetext{
* Corresponding author.

E-mail address: motaal@ufsj.edu.br (A.L. Mota).
} 
a hidden parameter which plays the role of renormalization group scale in renormalizable models. The imposition that the Green functions do not depend on how the infinities are subtracted from the amplitudes can be formulated by demanding that the Green functions are independent of such parameter. This gives rise to the running couplings, quantum mechanical mass generation, etc.

Recently Jackiw [1] raised an interesting matter concerning arbitrary parameters which appear in perturbative calculations in certain field theoretical models. Such arbitrary (regularizationdependent) parameters show up as differences between divergent integrals of the same superficial degree of divergence. Usually physicists do not like to have free parameters in their theoretical models. The prescription to deal with such arbitrary quantities is threefold: (1) check whether they may be considered as finite counterterms (as it is usual in renormalizable models); (2) check whether symmetry constraints (e.g. supersymmetry, gauge symmetry, etc.) may require that such parameters assume a definite value and (3) see if as a genuine free parameter, phenomenology can make use of it to fit the data in the underlying model. Of course such parameters are expected to play an essential role in finite theories and effective models.

A well-known example where a finite ambiguity plays an essential role is in the description of the Adler-Bardeen-Bell-Jackiw anomaly [2,3]. In this case the finite constant is a manifestation of an important symmetry breaking at the level of perturbative quantum corrections. A democratic description of the anomaly between the vector and axial sectors is achieved when we allow a regularization-dependent parameter to be arbitrary [4].

The ideal arena to implement such ideas in the momentum space is the implicit regularization (IR) scheme [5], which has already been successfully applied to several examples [6,4,7-9]. The idea behind IR is that one

(a) separates external momentum-independent divergencies from finite contributions, without the need of an explicit regulator;

(b) leaves their divergent content in the form of basic integrals until the end of the calculation;

(c) does not modify the underlying theory, preserving its symmetries and space-time dimension.

The purpose of the present contribution is the investigation in the LSM [12] of the role played by ambiguities in the context of the above discussed ideas. We show that radiative corrections yield together with well-known infinities also finite undetermined contributions. In this work we will follow Jackiw's suggestion and leave the arbitrary quantities open until the end of the calculations. Then, we will show that these undetermined contributions can be fixed by symmetry relations or absorbed by renormalization, in agreement with the ideas presented in [10]. From the point of view of the current phenomenology, because the present Lagrangian does not contain the important 't Hooft determinantal interaction [11], we restrict the fits to the pion and kaon sectors, which are not crucially dependent on it.

The work is organized as follows: in Section 2 the method is presented for the case of amplitudes containing different masses in the fermion propagators and we show the origin of ambiguities. The renormalization group scale is also introduced within IR. In Section 3 the model is renormalized and some ambiguities are shown to be absorbed by renormalization. Moreover the $\beta$-function is explicitly calculated and the renormalization group equation for the pion coupling constant is solved in Section 3.1. The position of the well-known Landau pole is identified and coincides with the position of the pole in the large Euclidean momentum region of the pion propagator. In Section 4 we derive the expressions for the weak decay constants $f_{\pi}$ and $f_{\kappa}$, as 
well as the electromagnetic form factor for the kaon. Numerical results are given in Section 5, and conclusions are given in Section 6.

\section{Treatment of divergent integrals, ambiguities and renormalization group scale}

In the present section we illustrate the relevant technical details of the renormalization procedure we use by working out explicitly a one-loop Feynman amplitude.

Consider the pseudoscalar amplitude with two quark masses $m_{u}$ and $m_{s}$

$$
\Pi^{\mathrm{PP}}=i \int_{\Lambda} \frac{d^{4} k}{(2 \pi)^{4}} \operatorname{Tr}\left(\gamma_{5} \frac{1}{\not k-m_{u}} \gamma_{5} \frac{1}{\not k-\not p-m_{s}}\right),
$$

where the symbol $\Lambda$ stands for a regulator which needs not be explicitated, but is necessary to give a meaning to $\Pi^{\mathrm{PP}}$. One is then allowed to algebraically manipulate the integrand. We do it in such a way that the divergencies appear as integrals and are separated from the finite (external momentum-dependent) contribution to Eq. (1). After taking the Dirac trace, we use the following algebraic identity

$$
\frac{1}{(k-p)^{2}-m^{2}}=\frac{1}{k^{2}-m^{2}}-\frac{p^{2}-2 p \cdot k}{\left[(p-k)^{2}-m^{2}\right]\left(k^{2}-m^{2}\right)}
$$

at the level of the integrand. Note that it allows one to confine the external momentum dependence in more convergent integrals. This relation should be used recursively until the finite part is completely separated from divergent integrals. We get

$$
\begin{aligned}
\Pi^{\mathrm{PP}}= & -2\left\{\int \frac{d^{4} k}{(2 \pi)^{4}} \frac{i}{k^{2}-m_{u}^{2}}+\int \frac{d^{4} k}{(2 \pi)^{4}} \frac{i}{(k-p)^{2}-m_{s}^{2}}\right. \\
& \left.+\left[\left(m_{s}-m_{u}\right)^{2}-p^{2}\right] \int \frac{d^{4} k}{(2 \pi)^{4}} \frac{i}{\left(k^{2}-m_{u}^{2}\right)\left[(k-p)^{2}-m_{s}^{2}\right]}\right\} .
\end{aligned}
$$

The first integral on the RHS is what we call a basic quadratic divergence

$$
I_{q}\left(m^{2}\right)=\int \frac{d^{4} k}{(2 \pi)^{4}} \frac{i}{k^{2}-m^{2}} .
$$

The second integral on the RHS is also a quadratic divergence, but it still possesses an external momentum dependence. If one uses Eq. (2), one sees that an arbitrariness emerges

$$
T\left(p^{2}, m_{i}\right)=\int \frac{d^{4} k}{(2 \pi)^{4}} \frac{i}{(k-p)^{2}-m_{s}^{2}}=I_{q}\left(m_{s}^{2}\right)+p^{\mu} p^{\nu} \Delta_{\mu \nu},
$$

where

$$
\Delta_{\mu \nu}=i \int \frac{d^{4} k}{(2 \pi)^{4}} \frac{4 k_{\mu} k_{\nu}}{\left(k^{2}-m_{s}^{2}\right)^{3}}-i \int \frac{d^{4} k}{(2 \pi)^{4}} \frac{g_{\mu \nu}}{\left(k^{2}-m_{s}^{2}\right)^{2}}=\alpha g_{\mu \nu},
$$

and $\alpha=$ const, i.e. the difference between two logarithmically divergent integrals. Eq. (6) is a surface term, a particular case of the general expression for surface terms (see derivation in Appendix A)

$$
S_{\mu_{1} \mu_{2} \ldots \mu_{2 n}}^{(A-2)}=i \int \frac{d^{2 \omega} p}{(2 \pi)^{4}}\left[\frac{g_{\mu_{1} \mu_{2} \ldots \mu_{2 n}}}{\left(p^{2}-m^{2}\right)^{A-n-1}}-\frac{2^{n} \Gamma(A-1)}{\Gamma(A-n-1)} \frac{p_{\mu_{1}} p_{\mu_{2}} \cdots p_{\mu_{2 n}}}{\left(p^{2}-m^{2}\right)^{A-1}}\right],
$$


where $\omega$ is a continuous dimension, $\mu_{i}$ is a Lorentz index and $g_{\mu_{1} \mu_{2} \ldots \mu_{2 n}}$ is the standard notation for symmetrized products of the $g_{\mu \nu}$ tensor. It is clear that at $\omega=2, A=4$ and $n=1$ we reproduce Eq. (6). In dimensional regularization or Pauli-Villars, following the usual prescriptions to preserve gauge invariance, one would get zero for $\Delta_{\mu \nu}$. However for other regularization prescriptions one may expect a dimensionless number. Following Jackiw's suggestion we leave this arbitrary number open until the end of the calculations and only then see if it must be fixed by Ward identities. In QED and QCD gauge invariance forces it to be zero [6]. In the anomalous pion decay, on the other hand, it must be finite [4].

The third term in Eq. (3) is also logarithmically divergent. In the spirit of implicit regularization the integral can be displayed as

$$
\begin{aligned}
\int \frac{d^{4} k}{(2 \pi)^{4}} \frac{i}{\left(k^{2}-m_{u}^{2}\right)\left[(k-p)^{2}-m_{s}^{2}\right]} & =\int \frac{d^{4} k}{(2 \pi)^{4}} \frac{i}{\left(k^{2}-m_{u}^{2}\right)^{2}}+F\left(m_{u}^{2}, m_{s}^{2}, p^{2} ; m_{u}^{2}\right) \\
& =\int \frac{d^{4} k}{(2 \pi)^{4}} \frac{i}{\left(k^{2}-m_{s}^{2}\right)^{2}}+F\left(m_{u}^{2}, m_{s}^{2}, p^{2} ; m_{s}^{2}\right) \\
& =\int \frac{d^{4} k}{(2 \pi)^{4}} \frac{i}{\left(k^{2}-\zeta^{2}\right)^{2}}+F\left(m_{u}^{2}, m_{s}^{2}, p^{2} ; \zeta^{2}\right),
\end{aligned}
$$

where again we separated another basic divergent integral

$$
I_{\log }\left(m^{2}\right)=\int \frac{d^{4} k}{(2 \pi)^{4}} \frac{i}{\left(k^{2}-m^{2}\right)^{2}},
$$

and a finite contribution

$$
F\left(m_{u}^{2}, m_{s}^{2}, p^{2} ; \zeta^{2}\right)=\frac{1}{(4 \pi)^{2}} \int_{0}^{1} d z \ln \left(\frac{p^{2} z(1-z)+\left(m_{u}^{2}-m_{s}^{2}\right) z-m_{u}^{2}}{-\zeta^{2}}\right) .
$$

In this expression the last argument corresponds to a squared mass in the denominator of the logarithm and plays the role of a scale. This comes from the property of the logarithmic divergence,

$$
I_{\log }\left(m^{2}\right)=I_{\log }\left(\zeta^{2}\right)+\frac{1}{(4 \pi)^{2}} \ln \left(\frac{m^{2}}{\zeta^{2}}\right),
$$

and its intimate connection with the finite part of the amplitude. The arbitrary scale $\zeta^{2}$ represents in this method the renormalization group scale.

Finally we obtain that

$$
\begin{aligned}
\Pi^{\mathrm{PP}}= & 2\left\{I_{q}\left(m_{u}^{2}\right)+I_{q}\left(m_{s}^{2}\right)+p^{2} \alpha\right. \\
& \left.+\left[\left(m_{u}-m_{s}\right)^{2}-p^{2}\right]\left(I_{\log }\left(\zeta^{2}\right)+F\left(m_{u}^{2}, m_{s}^{2}, p^{2} ; \zeta^{2}\right)\right)\right\} .
\end{aligned}
$$

Analogously one finds for the scalar amplitude

$$
\Pi^{\mathrm{SS}}=i \int_{\Lambda} \frac{d^{4} k}{(2 \pi)^{4}} \operatorname{Tr}\left(\frac{1}{\not k-m_{u}} \frac{1}{\not k-\not p-m_{s}}\right),
$$

the following relation

$$
\begin{aligned}
\Pi^{\mathrm{SS}}= & 2\left\{I_{q}\left(m_{u}^{2}\right)+I_{q}\left(m_{s}^{2}\right)+p^{2} \alpha\right. \\
& \left.+\left[\left(m_{u}+m_{s}\right)^{2}-p^{2}\right]\left(I_{\log }\left(\zeta^{2}\right)+F\left(m_{u}^{2}, m_{s}^{2}, p^{2} ; \zeta^{2}\right)\right)\right\} .
\end{aligned}
$$




\section{The model}

We start by considering the following generating functional

$$
Z=\int \prod_{a} \mathcal{D} \sigma_{0 a} \mathcal{D} \pi_{0 a} \mathcal{D} q \mathcal{D} \bar{q} \exp \left(i S\left(\bar{q}, q, \sigma_{0}, \pi_{0}\right)\right)
$$

with the action $S\left(\bar{q}, q, \sigma_{0}, \pi_{0}\right)$ of a $U(3) \times U(3)$ linear sigma model including fermionic degrees of freedom

$$
\begin{aligned}
& S\left(\bar{q}, q, \sigma_{0}, \pi_{0}\right)=\int d^{4} x\left[\mathcal{L}_{q}-\frac{\mu_{0}^{2}}{4} \operatorname{tr}\left(B B^{\dagger}\right)-\frac{\lambda_{0 q}}{2} \operatorname{tr}\left[\left(B B^{\dagger}\right)^{2}\right]+\frac{1}{2 g_{0}} \operatorname{tr}\left(c_{0} \sigma_{0}\right)\right. \\
& \left.+\frac{f_{0}^{2}}{4} \operatorname{tr}\left(\partial_{\mu} \sigma_{0} \partial^{\mu} \sigma_{0}+\partial_{\mu} \pi_{0} \partial^{\mu} \pi_{0}\right)\right], \\
& \mathcal{L}_{q}=\bar{q}\left[i \not \partial-g_{0}\left(\sigma_{0}+i \gamma_{5} \pi_{0}\right)\right] q .
\end{aligned}
$$

Here $B=\sigma_{0}+i \pi_{0}, B^{\dagger}=\sigma_{0}-i \pi_{0}$, used in the definition of the quadratic and quartic invariants in mesonic fields [13]

$$
\operatorname{tr}\left(B B^{\dagger}\right)=\operatorname{tr}\left(\sigma_{0}^{2}+\pi_{0}^{2}\right), \quad \operatorname{tr}\left[\left(B B^{\dagger}\right)^{2}\right]=\operatorname{tr}\left\{\left(\sigma_{0}^{2}+\pi_{0}^{2}\right)^{2}-\left[\sigma_{0}, \pi_{0}\right]^{2}\right\},
$$

with the scalar and pseudoscalar fields $\sigma_{0}=\lambda_{a} \sigma_{0}^{a}$ and $\pi_{0}=\lambda_{a} \pi_{0}^{a}$ containing the standard $U(3)$ matrices $\lambda_{a}(a=0,1, \ldots, 8)$ in flavor space. The subscript 0 stands for bare quantities, $\mathcal{L}_{q}$ describes the coupling of quark fields $q, \bar{q}$ to the mesons, $g_{0}$ is dimensionless.

The symmetry transformation properties of the fermionic fields are

$$
\delta q=i\left(\alpha^{\prime}+\gamma_{5} \beta^{\prime}\right) q, \quad \delta \bar{q}=-i \bar{q}\left(\alpha^{\prime}-\gamma_{5} \beta^{\prime}\right)
$$

and for the mesonic fields

$$
\delta \sigma_{0}=i\left[\alpha^{\prime}, \sigma_{0}\right]+\left\{\beta^{\prime}, \pi_{0}\right\}, \quad \delta \pi_{0}=i\left[\alpha^{\prime}, \pi_{0}\right]-\left\{\beta^{\prime}, \sigma_{0}\right\},
$$

where the parameters of the infinitesimal global transformations $\alpha^{\prime}$ and $\beta^{\prime}$ are Hermitian flavor matrices. The explicit symmetry breaking piece is introduced via the linear term proportional to $c_{0} \sigma_{0}$ with a diagonal matrix-valued strength $c_{0}=\operatorname{diag}\left\{c_{0 u}, c_{0 u}, c_{0 s}\right\}$. Without the quartic interaction and kinetic terms for the mesons, proportional to $f_{0}^{2}$ and $\lambda_{0 q}$ respectively, the action corresponds to a Nambu-Jona-Lasinio (NJL) type Lagrangian [14] in semi-bosonized form; this is achieved by the method of auxiliary fields [19], which has been widely used in various extensions of the original NJL Lagrangian, see e.g. [20-22]. We relate the $c_{0}$ to the diagonal current quark mass matrix $\hat{m}_{0}$ through the choice $c_{0}=\mu_{0}^{2} \hat{m}_{0}$. We restrict our study to the case $\hat{m}_{u}=\hat{m}_{d} \neq \hat{m}_{s}$, which breaks the unitary $S U(3)$ symmetry down to the subgroup $S U(2)_{I} \times U(1)_{Y}$ (isospinhypercharge). Upon the exact integration over the quark variables in the path integral Eq. (15), one obtains for the real part of the effective action associated with the fermions (we do not consider here anomalous processes),

$$
\int \mathcal{D} q \mathcal{D} \bar{q} \exp \left(i \int d^{4} x \mathcal{L}_{q}\right) \rightarrow \exp \left(\ln \left|\operatorname{det} D_{E}\right|\right)=\exp \left(\frac{1}{2} \operatorname{Tr} \ln \left(D_{E}^{\dagger} D_{E}\right)\right),
$$

where we use the strictly positive unbounded Hermitian second order elliptic operator $D_{E}^{\dagger} D_{E}$, which is chiral and gauge covariant [15-17] and where Tr designates functional trace, $D_{E}$ stands 
for the Euclidean Dirac operator

$$
D_{E}=i \gamma_{\mu} \partial_{\mu}-g_{0}\left(\sigma_{0}+i \gamma_{5} \pi_{0}\right), \quad D_{E}^{\dagger}=-i \gamma_{\mu} \partial_{\mu}-g_{0}\left(\sigma_{0}-i \gamma_{5} \pi_{0}\right) .
$$

Since the scalar fields possess non-vanishing vacuum expectation values, we perform the shift $\sigma_{0} \rightarrow \frac{m}{g_{0}}+\sigma_{0}$, where $m$ represents the finite constituent quark mass matrix and obtain after this shift

$$
D_{E}^{\dagger} D_{E}=m^{2}-\partial^{2}+Y,
$$

with the background mesonic fields [18]

$$
Y=i g_{0} \gamma_{\mu}\left(\partial_{\mu} \sigma_{0}+i \gamma_{5} \partial_{\mu} \pi_{0}\right)+g_{0}^{2}\left(\sigma_{0}^{2}+\left\{\frac{m}{g_{0}}, \sigma_{0}\right\}+\pi_{0}^{2}+i \gamma_{5}\left[\sigma_{0}+\frac{m}{g_{0}}, \pi_{0}\right]\right),
$$

leading to the expansion

$$
\begin{aligned}
\operatorname{Tr}\left(\ln D_{E}^{\dagger} D_{E}\right)= & \sum_{n=1}^{\infty} \frac{1}{n} \operatorname{Tr}\left\{( - \partial ^ { 2 } + m ^ { 2 } ) \left[1+\left(-\partial^{2}+m^{2}\right)^{-1}\left[i g_{0} \gamma_{\mu}\left(\partial_{\mu} \sigma_{0}+i \gamma_{5} \partial_{\mu} \pi_{0}\right)\right.\right.\right. \\
& \left.\left.\left.+g_{0}^{2}\left(\sigma_{0}^{2}+\left\{\sigma_{0}, m / g_{0}\right\}+\pi_{0}^{2}+i \gamma_{5}\left[\pi_{0}, \sigma_{0}+m / g_{0}\right]\right)\right]\right]\right\}^{n} .
\end{aligned}
$$

From now on it is convenient to use the following representation of the scalar and pseudoscalar fields

$$
\frac{\lambda_{a} \sigma_{a}}{\sqrt{2}}=\left(\begin{array}{ccc}
\frac{\sigma_{u}}{\sqrt{2}} & \sigma^{+} & \delta^{+} \\
\sigma^{-} & \frac{\sigma_{d}}{\sqrt{2}} & \delta^{0} \\
\delta^{-} & \bar{\delta}^{0} & \frac{\sigma_{s}}{\sqrt{2}}
\end{array}\right), \quad \frac{\lambda_{a} \pi_{a}}{\sqrt{2}}=\left(\begin{array}{ccc}
\frac{\phi_{u}}{\sqrt{2}} & \pi^{+} & K^{+} \\
\pi^{-} & \frac{\phi_{d}}{\sqrt{2}} & K^{0} \\
K^{-} & \bar{K}^{0} & \frac{\phi_{s}}{\sqrt{2}}
\end{array}\right) .
$$

The gap equation will be obtained by considering the $n=1$ term in the expansion equation (25) and the linear contributions in $\sigma_{0}$ from the remaining terms of Eq. (16) after the mass shift. We get

$$
\frac{\mu_{0}^{2}}{g_{0}^{2}} m_{i}-\frac{c_{0 i}}{g_{0}^{2}}-8 N_{c} m_{i} I_{q}\left(m_{i}\right)+2 \frac{\lambda_{0 q}}{g_{0}^{4}} m_{i}^{3}=0,
$$

where $\{i=u, s\}$.

The $n=2$ term in the expansion contains all the other divergent contributions which go up to four-point functions. In the calculations there appear the two basic divergent integrals $I_{q}$ and $I_{\log }$, Eqs. (4) and (9), as well as the difference between two logarithmically divergent integrals, $\alpha$, Eq. (6). Expressions (12) and (14), after properly taking into account $g_{0}$ and trace factors in color $\left(N_{c}\right)$ and Dirac spaces, are the amplitudes, continued to Minkowski space, resulting from terms quadratic in the fields of (25).

In the following we need the wave function renormalizations, which are readily obtained as the coefficients of the $p^{2}$ terms in all expressions quadratic in the fields, stemming from the kinetic terms proportional to $f_{0}^{2}$ and from the amplitudes (12), (14)

$$
\begin{aligned}
Z_{\sigma}^{-1}\left(m_{i}, m_{j} ; \zeta^{2}\right)= & f_{0}^{2}-4 N_{c} g_{0}^{2}\left\{I_{\log }\left(\zeta^{2}\right)-\alpha+F\left(m_{i}, m_{j}, 0 ; \zeta^{2}\right)\right. \\
& \left.-\left(m_{i}+m_{j}\right)^{2} F^{\prime}\left(m_{i}, m_{j}, 0 ; \zeta^{2}\right)\right\}, \\
Z_{\pi}^{-1}\left(m_{i}, m_{j} ; \zeta^{2}\right)= & f_{0}^{2}-4 N_{c} g_{0}^{2}\left\{I_{\log }\left(\zeta^{2}\right)-\alpha+F\left(m_{i}, m_{j}, 0 ; \zeta^{2}\right)\right. \\
& \left.-\left(m_{i}-m_{j}\right)^{2} F^{\prime}\left(m_{i}, m_{j}, 0 ; \zeta^{2}\right)\right\}
\end{aligned}
$$


where $F^{\prime}$ represents the derivative with respect to square external momentum of $F$, taken at $p^{2}=0$. The retained finite terms $F\left(m_{i}, m_{j}, 0 ; \zeta^{2}\right)$ and $F^{\prime}\left(m_{i}, m_{j}, 0 ; \zeta^{2}\right)$ lead to different wave function renormalizations for the different members of the pseudoscalar and scalar nonets; the higher contributions to the expansion of $F\left(m_{i}, m_{j}, p^{2} ; \zeta^{2}\right)$ will be absorbed in the renormalized propagators of the mesons (see Eq. (42) below).

The renormalized coupling constants $g_{\sigma}\left(m_{i}, m_{j}\right)$ and $g_{\pi}\left(m_{i}, m_{j}\right)$ are then obtained as

$$
\begin{aligned}
& \frac{g_{0}^{2}}{g_{\sigma}^{2}\left(m_{i}, m_{j} ; \zeta^{2}\right)}=Z_{\sigma}^{-1}\left(m_{i}, m_{j} ; \zeta^{2}\right), \\
& \frac{g_{0}^{2}}{g_{\pi}^{2}\left(m_{i}, m_{j} ; \zeta^{2}\right)}=Z_{\pi}^{-1}\left(m_{i}, m_{j} ; \zeta^{2}\right),
\end{aligned}
$$

and the renormalized masses $\mu_{\sigma, \pi}\left(m_{i}, m_{j} ; \zeta^{2}\right)$ become

$$
\begin{aligned}
\frac{\mu_{\sigma, \pi}^{2}\left(m_{i}, m_{j} ; \zeta^{2}\right)}{Z_{\sigma, \pi}\left(m_{i}, m_{j} ; \zeta^{2}\right)}= & M_{\sigma, \pi}^{2}\left(m_{i}, m_{j} ; \zeta^{2}\right) \\
= & \mu_{0}^{2}-4 N_{c} g_{0}^{2}\left(I_{q}\left(m_{i}^{2}\right)+I_{q}\left(m_{j}^{2}\right)\right) \\
& -4 N_{c} g_{0}^{2}\left(m_{i} \pm m_{j}\right)^{2}\left(I_{\log }\left(\zeta^{2}\right)+F\left(m_{i}, m_{j}, 0 ; \zeta^{2}\right)\right) \\
& +2 \frac{\lambda_{0 q}}{g_{0}^{2}}\left(m_{i}^{2}+m_{j}^{2} \pm m_{i} m_{j}\right),
\end{aligned}
$$

where $M_{\sigma}^{2}$ goes with the plus signs, and the renormalized quartic coupling

$$
\frac{\lambda_{q}}{g_{\pi}^{2}\left(m_{u}, m_{u} ; \zeta^{2}\right)}=\frac{\lambda_{0 q}}{g_{0}^{4}}-4 N_{c} I_{\log }\left(\zeta^{2}\right) .
$$

We have retained in the renormalization of the masses the finite terms $F\left(m_{i}, m_{j}, 0 ; \zeta^{2}\right)$. Note that all ambiguities $\alpha$ appear in the field renormalization coefficients (28), (29). In principle they could assume different values for the different processes. Chiral symmetry restricts them to have the same value, as we shall see below. Using relations (31) and (32) one obtains that the kaon and pion renormalization constants are related as (where we have identified $g_{\pi}=g_{\pi}\left(m_{u}, m_{u} ; \zeta^{2}\right.$ ) and $\left.g_{\kappa}=g_{\pi}\left(m_{u}, m_{s} ; \zeta^{2}\right)\right)$

$$
\begin{aligned}
& \frac{1}{g_{\kappa}^{2}}= \frac{1}{g_{\pi}^{2}}-4 N_{c}\left[F\left(m_{u}, m_{s}, 0 ; \zeta^{2}\right)-F\left(m_{u}, m_{u}, 0 ; \zeta^{2}\right)\right. \\
&\left.-\left(m_{u}-m_{s}\right)^{2} F^{\prime}\left(m_{u}, m_{s}, 0 ; \zeta^{2}\right)\right], \\
& \frac{\mu_{\kappa}^{2}}{g_{\kappa}^{2}}= \frac{\mu_{\pi}^{2}}{g_{\pi}^{2}}+2 m_{s}\left(m_{s}-m_{u}\right)\left[\frac{\lambda_{q}}{g_{\pi}^{2}}-4 N_{c} F\left(m_{u}, m_{s}, 0 ; \zeta^{2}\right)\right], \\
& F\left(m_{u}, m_{s}, 0 ; \zeta^{2}\right)=-\frac{1}{(4 \pi)^{2}}\left[\frac{m_{s}^{2}}{m_{s}^{2}-m_{u}^{2}} \ln \left(\frac{m_{s}^{2}}{m_{u}^{2}}\right)+\ln \left(\frac{m_{u}^{2}}{\zeta^{2}}\right)-1\right] .
\end{aligned}
$$

With these definitions we obtain the effective action

$$
S=S_{\text {kin }}+S_{\text {mass }}+S_{\text {int }},
$$

where $S_{\text {kin }}, S_{\text {mass }}, S_{\text {int }}$ are the actions related to kinetic, mass and interaction terms of the mesons (in the equations for $S_{\text {kin }}, S_{\text {mass }}$ below we omit the index 0 in the bare fields in order not to clutter the notation). 


$$
\begin{aligned}
S_{\text {kin }}= & \int d^{4} x\left\{Z_{\sigma}^{-1}\left(m_{u}, m_{u}\right)\left(\partial_{\mu} \sigma_{u} \partial^{\mu} \sigma_{u}+\partial_{\mu} \sigma_{d} \partial^{\mu} \sigma_{d}+\partial_{\mu} \sigma^{+} \partial^{\mu} \sigma^{-}\right)\right. \\
& +Z_{\sigma}^{-1}\left(m_{s}, m_{s}\right) \partial_{\mu} \sigma_{s} \partial^{\mu} \sigma_{s}+Z_{\sigma}^{-1}\left(m_{u}, m_{s}\right)\left(\partial_{\mu} \delta^{+} \partial^{\mu} \delta^{-}+\partial_{\mu} \delta^{0} \partial^{\mu} \bar{\delta}^{0}\right) \\
& +Z_{\pi}^{-1}\left(m_{u}, m_{u}\right)\left(\partial_{\mu} \phi_{u} \partial^{\mu} \phi_{u}+\partial_{\mu} \phi_{d} \partial^{\mu} \phi_{d}+\partial_{\mu} \pi^{+} \partial^{\mu} \pi^{-}\right) \\
& \left.+Z_{\pi}^{-1}\left(m_{s}, m_{s}\right) \partial_{\mu} \phi_{s} \partial^{\mu} \phi_{s}+Z_{\pi}^{-1}\left(m_{u}, m_{s}\right)\left(\partial_{\mu} K^{+} \partial^{\mu} K^{-}+\partial_{\mu} K^{0} \partial^{\mu} \bar{K}^{0}\right)\right\}, \\
S_{\text {mass }}= & -\int d^{4} x\left\{M_{\sigma}^{2}\left(m_{u}, m_{u}\right)\left(\sigma_{u}^{2}+\sigma_{d}^{2}+\sigma^{+} \sigma^{-}\right)+M_{\sigma}^{2}\left(m_{s}, m_{s}\right) \sigma_{s}^{2}\right. \\
& +M_{\sigma}^{2}\left(m_{u}, m_{s}\right)\left(\delta^{0} \bar{\delta}^{0}+\delta^{+} \delta^{-}\right)+M_{\pi}^{2}\left(m_{u}, m_{u}\right)\left(\phi_{u}^{2}+\phi_{d}^{2}+\pi^{+} \pi^{-}\right) \\
& \left.+M_{\pi}^{2}\left(m_{s}, m_{s}\right) \phi_{s}^{2}+M_{\pi}^{2}\left(m_{u}, m_{s}\right)\left(K^{0} \bar{K}^{0}+K^{+} K^{-}\right)\right\} .
\end{aligned}
$$

If we use the gap equations in order to eliminate the quadratic divergencies in the expressions for the renormalized masses $\mu_{\sigma, \pi}^{2}\left(m_{i}, m_{j}\right)$, Eq. (32), it is possible to define the renormalized coupling $C_{i}$ through

$$
\begin{aligned}
& C_{u}=\frac{c_{u 0}}{g_{0}^{2}}=m_{u} \frac{\mu_{\pi}^{2}}{g_{\pi}^{2}}, \\
& C_{s}=\frac{c_{s 0}}{g_{0}^{2}}=m_{s}\left(\frac{2 \mu_{\kappa}^{2}}{g_{\kappa}^{2}}-\frac{\mu_{\pi}^{2}}{g_{\pi}^{2}}-2\left(m_{u}-m_{s}\right)^{2}\left(\frac{\lambda_{q}}{g_{\pi}^{2}}-4 N_{c} F\left(m_{u}, m_{s}, 0 ; \zeta^{2}\right)\right)\right) .
\end{aligned}
$$

Using (35) one obtains also

$$
C_{u}+C_{s}=\left(m_{u}+m_{s}\right) \frac{\mu_{\kappa}^{2}}{g_{\kappa}^{2}} .
$$

Now we write out the renormalized propagators for pions and kaons, since they will be used in the remaining,

$$
\begin{aligned}
& \Delta_{\pi}^{-1}\left(p^{2}\right)=p^{2}-\mu_{\pi}^{2}+4 N_{c} g_{\pi}^{2} F_{\mathrm{fin}}\left(m_{u}, m_{u}, p^{2} ; \zeta^{2}\right), \\
& \Delta_{\kappa}^{-1}\left(p^{2}\right)=p^{2}-\mu_{\kappa}^{2}+4 N_{c} g_{\kappa}^{2} \Sigma_{\mathrm{fin}}\left(m_{u}, m_{s}, p^{2} ; \zeta^{2}\right)
\end{aligned}
$$

with the finite momentum-dependent contributions

$$
F_{\text {fin }}\left(m_{u}, m_{u}, p^{2} ; \zeta^{2}\right)=-p^{2}\left(F\left(m_{u}, m_{u}, p^{2} ; \zeta^{2}\right)-F\left(m_{u}, m_{u}, 0 ; \zeta^{2}\right)\right),
$$

where in particular $F\left(m_{i}, m_{i}, 0 ; m_{i}\right)=0$,

$$
\begin{aligned}
\Sigma_{\mathrm{fin}}\left(m_{u}, m_{s}, p^{2} ; \zeta^{2}\right)= & {\left[\left(m_{s}-m_{u}\right)^{2}-p^{2}\right]\left\{F\left(m_{u}, m_{s}, p^{2} ; \zeta^{2}\right)-F\left(m_{u}, m_{s}, 0 ; \zeta^{2}\right)\right\} } \\
& -p^{2}\left(m_{s}-m_{u}\right)^{2} F^{\prime}\left(m_{u}, m_{s}, 0 ; \zeta^{2}\right),
\end{aligned}
$$

where use has been made of the normalization conditions

$$
\Delta_{\pi, \kappa}^{-1}(0)=-\mu_{\pi, \kappa}^{2},\left.\quad \frac{d \Delta_{\pi, \kappa}^{-1}\left(p^{2}\right)}{d p^{2}}\right|_{p^{2}=0}=1 .
$$

We obtain finally the physical pseudoscalar masses as zeros of these propagators

$$
\begin{aligned}
& m_{\pi}^{2}=\mu_{\pi}^{2}-4 N_{c} g_{\pi}^{2} F_{\mathrm{fin}}\left(m_{u}, m_{u}, m_{\pi}^{2} ; \zeta^{2}\right), \\
& m_{\kappa}^{2}=\mu_{\kappa}^{2}-4 N_{c} g_{\kappa}^{2} \Sigma_{\mathrm{fin}}\left(m_{u}, m_{s}, m_{\kappa}^{2} ; \zeta^{2}\right) .
\end{aligned}
$$


At the physical meson masses we obtain the following pseudoscalar quark couplings

$$
\begin{aligned}
& g_{\pi q q}^{-2}=g_{\pi}^{-2}+\left.4 N_{c} \frac{d F_{\mathrm{fin}}\left(m_{u}, m_{s}, p^{2} ; \zeta^{2}\right)}{d p^{2}}\right|_{p^{2}=m_{\pi}^{2}}, \\
& g_{\kappa q q}^{-2}=g_{\kappa}^{-2}+\left.4 N_{c} \frac{d \Sigma_{\mathrm{fin}}\left(m_{u}, m_{s}, p^{2} ; \zeta^{2}\right)}{d p^{2}}\right|_{p^{2}=m_{\kappa}^{2}} .
\end{aligned}
$$

\subsection{The $\beta$-function}

In order to illustrate the role of the arbitrary scale $\zeta^{2}$ introduced in the previous sections, we evaluate the $\beta$-function for the pseudoscalars

$$
\beta_{\pi}=\zeta \frac{\partial}{\partial \zeta} g_{\pi}\left(\zeta^{2}\right)
$$

Using Eq. (31) we get to one-loop order

$$
\beta_{\pi}=\frac{N_{c} g_{\pi}^{3}}{4 \pi^{2}}
$$

From the renormalization group equations (49) and (50) one solves for $g_{\pi}$ and gets

$$
g_{\pi}^{2}\left(\zeta^{\prime 2}\right)=\frac{g_{\pi}^{2}\left(\zeta^{2}\right)}{1-\frac{N_{c}}{4 \pi^{2}} g_{\pi}^{2}\left(\zeta^{2}\right) \ln \left(\frac{\zeta^{\prime 2}}{\zeta^{2}}\right)} .
$$

Analogously to QED at one-loop level, a Landau pole appears in Eq. (51) and it corresponds to a pole in the large Euclidean momentum region of the pion propagator. This is easily seen by expanding for large negative $p^{2}$ the inverse of the renormalized pion propagator, Eq. (42)

$$
\Delta_{\pi}^{-1} \sim\left|p^{2}\right|\left(\frac{N_{c}}{4 \pi^{2}} g_{\pi}^{2} \ln \frac{\left|p^{2}\right|}{\zeta^{2}}-1\right)+\cdots .
$$

\section{Coupling to external fields}

In order to make contact to phenomenology an important issue is the calculation of electromagnetic form factors and weak decay constants. We treat the vector and axial currents as external classical fields. We extract these quantities from a generalized expression for the Dirac operator, now containing the vector $V_{\mu}$ and axialvector $A_{\mu}$ fields. The previous expression for $D^{\dagger} D$ is now replaced by

$$
\begin{aligned}
D_{E}^{\dagger} D_{E}= & m^{2}-d_{\mu}^{2}+i g_{0} \gamma_{\mu}\left(\Delta_{\mu} \sigma_{0}-i \gamma_{5} \Delta_{\mu} \pi_{0}\right)-\frac{i}{4}\left[\gamma_{\mu}, \gamma_{\nu}\right] \Gamma_{\mu \nu} \\
& +g_{0}^{2}\left(\sigma_{0}^{2}+\left\{\sigma_{0}, m / g_{0}\right\}+\pi_{0}^{2}+i \gamma_{5}\left[\sigma_{0}+m / g_{0}, \pi_{0}\right]\right),
\end{aligned}
$$

where

$$
-d_{\mu}^{2}=-\partial_{\mu}^{2}+2 i \Gamma_{\mu} \partial_{\mu}+i \partial_{\mu} \Gamma_{\mu}+\Gamma_{\mu}^{2}
$$

and $\Gamma_{\mu}=V_{\mu}+\gamma_{5} A_{\mu}, \Gamma_{\mu \nu}=V_{\mu \nu}+\gamma_{5} A_{\mu \nu}$. The covariant derivatives are 


$$
\begin{aligned}
& \nabla_{\mu} \sigma_{0}=\partial_{\mu} \sigma_{0}+\left\{A_{\mu}, \pi_{0}\right\}-i\left[V_{\mu}, \sigma_{0}+m / g_{0}\right], \\
& \nabla_{\mu} \pi_{0}=\partial_{\mu} \pi_{0}-\left\{A_{\mu}, \sigma_{0}+m / g_{0}\right\}-i\left[V_{\mu}, \pi_{0}\right], \\
& V_{\mu \nu}=\partial_{\mu} V_{\nu}-\partial_{\nu} V_{\mu}-i\left[A_{\mu}, A_{\nu}\right]-i\left[V_{\mu}, V_{\nu}\right], \\
& A_{\mu \nu}=\partial_{\mu} A_{\nu}-\partial_{\nu} A_{\mu}-i\left[V_{\mu}, A_{\nu}\right]-i\left[A_{\mu}, V_{\nu}\right] .
\end{aligned}
$$

The calculation of $f_{\pi}$ and $f_{\kappa}$ to one-loop order is now straightforward, after collecting the relevant vertices involving one pseudoscalar and one axialvector fields. Since we are treating the external currents classically, we disregard all quantum dynamical corrections to the vector fields. Also the kinetic terms proportional to $f_{0}^{2}$ involving the scalar and pseudoscalar fields in Eq. (16) have to be minimally coupled to the external currents through the covariant derivatives Eq. (55). We obtain the bare $f_{\pi}^{0}$ as

$$
\begin{aligned}
f_{\pi}^{0}\left(m_{\pi}^{2}\right) & =\frac{m_{u}}{g_{0}}\left(-4 N_{c} g_{0}^{2} I_{\log }\left(\zeta^{2}\right)+f_{0}^{2}-4 N_{c} g_{0}^{2} F\left(m_{u}, m_{u}, m_{\pi}^{2} ; \zeta^{2}\right)\right) \\
& =\frac{m_{u}}{g_{0}} Z_{\pi}^{-1}\left(m_{u}, m_{u}\right)\left(1-4 N_{c} g_{\pi}^{2}\left(\alpha-\frac{F_{\mathrm{fin}}\left(m_{u}, m_{u}, m_{\pi}^{2} ; \zeta^{2}\right)}{m_{\pi}^{2}}\right)\right) .
\end{aligned}
$$

In order to obtain the Goldberger-Treiman relation of chiral symmetry it is necessary to avoid the ambiguous $\alpha$ term present in Eq. (59). This can be done by either choosing $\alpha=0$ at this point [23] or by introducing the following counterterms

$$
\begin{aligned}
L_{\mathrm{ct}}= & \frac{f^{2} g_{0}^{2}}{4} \operatorname{Tr}\left(-\partial_{\mu} \pi\left\{A_{\mu}, \sigma_{0}+\frac{m}{g_{0}}\right\}-i \partial_{\mu} \pi_{0}\left[V_{\mu}, \pi\right]\right. \\
& \left.+\partial_{\mu} \sigma_{0}\left\{A_{\mu}, \pi_{0}\right\}-i \partial_{\mu} \sigma_{0}\left[V_{\mu}, \sigma_{0}+\frac{m}{g_{0}}\right]\right),
\end{aligned}
$$

with a parameter $f^{2}$ to be fixed appropriately. This is the minimal chiral and gauge invariant combination available which contains a term that can absorb the $\alpha$ ambiguity. For the present purpose this is enough, although one might need to consider higher than cubic field interaction terms as well to realize the complete renormalization program. Note that this counterterm has consequences for the scalar-vector (SV) sector, which will be discussed below. Including the terms from (60) that contribute to the pion weak decay constant (proportional to $A_{\mu} \partial^{\mu} \pi$ ), we obtain

$$
f_{\pi}^{0}\left(m_{\pi}^{2}\right)=\frac{m_{u}}{g_{0}} Z_{\pi}^{-1}\left(m_{u}, m_{u}\right)\left(1+f^{2} g_{\pi}^{2}-4 N_{c} g_{\pi}^{2}\left(\alpha-\frac{F_{\mathrm{fin}}\left(m_{u}, m_{u}, m_{\pi}^{2} ; \zeta^{2}\right)}{m_{\pi}^{2}}\right)\right) .
$$

Using that

$$
-\frac{4 N_{c} g_{\pi}^{2}}{m_{\pi}^{2}} F_{\mathrm{fin}}\left(m_{u}, m_{u}, m_{\pi}^{2} ; \zeta^{2}\right)=1-\frac{\mu_{\pi}^{2}}{m_{\pi}^{2}}
$$

and the relation between the physical and bare pion field at the physical pion mass, $\pi=\pi^{\mathrm{ph}} \frac{g_{\pi q q}}{g_{0}}$, we get the renormalized weak pion decay constant

$$
f_{\pi}\left(m_{\pi}^{2}\right)=m_{u} \frac{g_{\pi q q}}{g_{\pi}^{2}} \frac{\mu_{\pi}^{2}}{m_{\pi}^{2}}
$$

provided

$$
f^{2}=4 N_{c} \alpha
$$

in Eq. (61), as a consequence of requiring chiral symmetry. 
Now, for the kaon weak decay constant one obtains

$$
\begin{aligned}
f_{\kappa}^{0}\left(m_{\kappa}^{2}\right)= & 2 N_{c} \frac{g_{0}}{m_{\kappa}^{2}}\left(m_{s}-m_{u}\right)\left[\left(I_{q}\left(m_{u}^{2}\right)-I_{q}\left(m_{s}^{2}\right)+m_{\kappa}^{2} \alpha\right)\right. \\
& \left.+\left(m_{s}^{2}-m_{u}^{2}\right)\left(I_{\log }\left(\zeta^{2}\right)+F\left(m_{u}, m_{s}, m_{\kappa}^{2} ; \zeta^{2}\right)\right)\right] \\
& +\frac{\left(m_{u}+m_{s}\right)}{2 g_{0}}\left[-4 N_{c} g_{0}^{2} I_{\log }\left(\zeta^{2}\right)+f_{0}^{2}+f^{2} g_{0}^{2}\right. \\
& \left.-4 N_{c} g_{0}^{2} F\left(m_{u}, m_{s}, m_{\kappa}^{2} ; \zeta^{2}\right)\right] .
\end{aligned}
$$

The term proportional to $\left(m_{u}+m_{S}\right)$ is the term which in the limit of equal masses reproduces $f_{\pi}$. We see that the introduction of the counterterms (60) in the model Lagrangian, with $f^{2}=4 N_{c} \alpha$ allows the $\left(m_{u}+m_{S}\right)$ part of (65) to be renormalized in the same way we did for $f_{\pi}$. However there remains an $\alpha$-dependent piece, proportional to the difference of the quark masses, which cannot be removed by the counterterm, nor by renormalization. It is interesting to note that the ambiguity $\alpha$ can neither be fixed by the Ward identity related to the pseudoscalar/axialvector radiative amplitude

$$
p^{\mu} \Pi_{\mu}^{\mathrm{PA}}=\left(m_{u}+m_{s}\right) \Pi^{\mathrm{PP}}+4 m_{u} T\left(p^{2}, m_{u}\right)+4 m_{s} I_{q}\left(m_{s}^{2}\right),
$$

where

$$
\Pi_{\mu}^{\mathrm{PA}}=i \int_{\Lambda} \frac{d^{4} k}{(2 \pi)^{4}} \operatorname{Tr}\left(\gamma_{5} \frac{1}{k-m_{s}} \gamma_{\mu} \gamma_{5} \frac{1}{k-\not p-m_{u}}\right)
$$

and $T\left(p^{2}, m_{i}\right)$ given in Eq. (5). One can verify that Eq. (66) is fulfilled without fixing $\alpha$, as long as the ambiguity present in the pseudoscalar/axialvector amplitude and the one present in the pseudoscalar/pseudoscalar amplitude are the same. One concludes that in the PA sector alone an ambiguity remains undetermined, which is compatible with the Goldberger-Treiman relation and modifies the PCAC relation only as a next to leading order correction in the symmetry breaking term and is leading correction in flavor symmetry breaking. This conclusion is however fallacious, as the chiral symmetry partner sector SV has to be analyzed in parallel. By fixing $\alpha$ through Eq. (64), Eq. (60) introduces counterterms in the scalar-vector sector

$$
L_{\mathrm{ctSV}}=\left(m_{s}-m_{u}\right) f^{2}\left(V_{\mu}^{+} \partial_{\mu} \delta^{-}-V_{\mu}^{-} \partial_{\mu} \delta^{+}+V_{\mu}^{\prime+} \partial_{\mu} \bar{\delta}_{0}-V_{\mu}^{\prime-} \partial_{\mu} \delta_{0}\right),
$$

where $V_{\mu}^{+}=V_{4 \mu}-i V_{5 \mu}, V_{\mu}^{-}=V_{4 \mu}+i V_{5 \mu}, V_{\mu}^{\prime+}=V_{6 \mu}-i V_{7 \mu}$ and $V_{\mu}^{\prime-}=V_{6 \mu}+i V_{7 \mu}$. These counterterms absorb the ambiguous $\alpha$ proportional terms which are generated radiatively through

$$
\Pi_{\mathrm{SV}}^{\mu, a b}=\frac{i}{4} \int \frac{d^{4} k}{(2 \pi)^{4}} \operatorname{Tr}\left(\frac{1}{k-m} \gamma^{\mu} \lambda_{a} \frac{1}{k-\not p-m} \lambda_{b}\right) .
$$

However, opposite to the PA case, these counterterms are proportional to the difference of the quark masses and will leave untouched an ambiguous term proportional to the sum of quark masses. This latter appears in a similar fashion as in Eq. (65) through the difference of quadratic divergencies with different external momentum dependence. This term violates chiral symmetry, since the SV amplitude must vanish in the limit of equal quark masses. We conclude therefore that the ambiguity $\alpha$ must be set to zero in this sector. This immediately implies that it must be zero as well in the PA sector, as the counterterms in the two sectors are linked through chiral symmetry.

We consider from now on the case with $\alpha=0$. 
Returning to Eq. (65), we observe that the quadratic divergencies can be removed through the gap equations and the other divergencies by renormalization. Using Eqs. (34)-(36) and (47) $f_{\kappa}$ reduces to

$$
f_{\kappa}\left(m_{\kappa}^{2}\right)=g_{\kappa q q} \frac{\left(m_{u}+m_{s}\right)}{2} \frac{\mu_{\kappa}^{2}}{m_{\kappa}^{2} g_{\kappa}^{2}} .
$$

At this stage it is useful to see how the expressions for $f_{\pi}$ and $f_{\kappa}$ comply with PCAC. For that we use the explicit symmetry breaking term of the Lagrangian in Eq. (16), [13]

$$
\delta \mathcal{L}_{\mathrm{SB}}=\frac{1}{2 g_{0}} \delta \operatorname{Tr}\left(c_{0} \sigma_{0}\right)
$$

to obtain for instance for one isotopic component of the pion field

$$
\frac{\partial \delta \mathcal{L}_{\mathrm{SB}}}{\partial \beta_{1}}=-2 \pi_{1} \frac{C_{0 u}}{g_{0}}=-2 \pi_{1}^{\mathrm{ph}} g_{\pi q q} C_{u}
$$

and for one flavor component of the kaon

$$
\frac{\partial \delta \mathcal{L}_{\mathrm{SB}}}{\partial \beta_{4}}=-K_{4} \frac{C_{0 u}+C_{0 s}}{g_{0}}=-K_{4}^{\mathrm{ph}} g_{K q q}\left(C_{u}+C_{s}\right) .
$$

Upon using here Eqs. (40) and (41) and the expressions (63) and (70) one obtains

$$
\begin{aligned}
& \frac{\partial \delta \mathcal{L}_{\mathrm{SB}}}{\partial \beta_{1}}=-2 \pi_{1}^{\mathrm{ph}} m_{\pi}^{2} f_{\pi}\left(m_{\pi}^{2}\right), \\
& \frac{\partial \delta \mathcal{L}_{\mathrm{SB}}}{\partial \beta_{4}}=-2 K_{4}^{\mathrm{ph}} m_{\kappa}^{2} f_{\kappa}\left(m_{\kappa}^{2}\right) .
\end{aligned}
$$

So, the pion and the kaon fulfill exactly PCAC.

\section{Numerical results}

In this section, we fit the model parameters to reproduce the pion and kaon masses, as well as the pion weak decay constant, in order to obtain a numerical estimative to the kaon weak decay constant and the kaon electromagnetic form factor. The model parameters are fixed by means of Eq. (47) and (59) in order to reproduce $m_{\pi}=139 \mathrm{MeV}, m_{\kappa}=494 \mathrm{MeV}$ and $f_{\pi}=93.3 \mathrm{MeV}$, with the renormalization constants $g_{\kappa}^{2}$ and $\mu_{\kappa}^{2}$ given by (34) and (35).

As pointed in Ref. [24], the action defined by (37) gives the same formal results as the conventional NJL model (with a $\Lambda \rightarrow \infty$ cut-off) in the case where $\lambda_{q}=1$. In particular, the Nambu relation in the chiral limit $m_{\sigma}=2 m$ holds only in the $\lambda_{q}=1$ case. We will proceed the parameters fitting with $\lambda_{q}=1$, and will return to this point latter. We also use $m_{u}=210 \mathrm{MeV}$ and $m_{u}=350 \mathrm{MeV}$, values largely employed on literature, generating the two set of parameters showed on Table 1.

With these choices for the parameters, and with $N_{c}=3$, we obtain for the kaon weak decay constant, $f_{\kappa}=114.2 \mathrm{MeV}$ (for $m_{u}=210 \mathrm{MeV}$ ) and $f_{\kappa}=71.6 \mathrm{MeV}$ (for $m_{u}=350 \mathrm{MeV}$ ).

The value of $f_{\kappa}$ estimated by the set of parameters corresponding to $m_{u}=210 \mathrm{MeV}$ is only $\sim 3 \%$ lower than the experimental results. Nevertheless, for $m_{u}=350 \mathrm{MeV}$ the kaon weak decay constant is still away from its experimental value, as already known.

We also evaluate the pion and kaon electromagnetic form factors, and the corresponding pion and kaon charge radius in the space-like region, given by 
Table 1

Parameters of the model, fitted to reproduce $m_{\pi}=139 \mathrm{MeV}, m_{\kappa}=494 \mathrm{MeV}$ and $f_{\pi}=93.3 \mathrm{MeV}$, with $m_{u}=210 \mathrm{MeV}$ and $m_{u}=350 \mathrm{MeV}$

\begin{tabular}{lll}
\hline Set & $m_{u}=210 \mathrm{MeV}$ & $m_{u}=350 \mathrm{MeV}$ \\
\hline$g_{\pi}$ & 2.250 & 3.752 \\
$\mu_{\pi}$ & $141.1 \mathrm{MeV}$ & $141.0 \mathrm{MeV}$ \\
$m_{S}$ & $479.0 \mathrm{MeV}$ & $601.7 \mathrm{MeV}$ \\
$f_{\kappa}$ & $114.0 \mathrm{MeV}$ & $71.6 \mathrm{MeV}$ \\
$\left\langle r_{\kappa}^{2}\right\rangle^{1 / 2}$ & $0.573 \mathrm{fm}$ & $0.608 \mathrm{fm}$ \\
\hline
\end{tabular}

$$
\begin{aligned}
F_{\kappa}= & -N_{c} g_{\kappa q q}^{2}\left[\frac{1}{g_{\pi}^{2}}+F\left(m_{u}^{2}, m_{u}^{2}, q^{2} ; \zeta^{2}\right)-\left(m_{\kappa}^{2}-\left(m_{s}-m_{u}\right)^{2}\right) F^{\prime}\left(m_{s}, m_{u}, m_{\kappa}^{2} ; \zeta^{2}\right)\right. \\
& \left.+I_{t}\left(q^{2}, m_{s}, m_{u}\right)\right]-N_{c} g_{\kappa q q}^{2}\left[\frac{1}{g_{\pi}^{2}}+F\left(m_{s}^{2}, m_{s}^{2}, q^{2} ; \zeta^{2}\right)\right. \\
& \left.-\left(m_{\kappa}^{2}-\left(m_{s}-m_{u}\right)^{2}\right) F^{\prime}\left(m_{u}, m_{s}, m_{\kappa}^{2} ; \zeta^{2}\right)+I_{t}\left(q^{2}, m_{u}, m_{s}\right)\right],
\end{aligned}
$$

and

$$
\left\langle r_{\kappa}^{2}\right\rangle=-\left.6 \frac{d F_{\kappa}\left(Q^{2}\right)}{d Q^{2}}\right|_{Q^{2} \rightarrow 0}
$$

where

$$
I_{t}\left(q^{2}, m_{u}, m_{s}\right)=i \int \frac{d^{4} k}{(2 \pi)^{4}} \frac{1}{\left(k^{2}-m_{u}^{2}\right)\left[\left(k-k_{1}\right)^{2}-m_{s}^{2}\right]\left[\left(k-k_{2}\right)^{2}-m_{s}^{2}\right]}
$$

with

$$
k_{1}^{2}=k_{2}^{2}=m_{\kappa}^{2}
$$

and

$$
k_{1} \cdot k_{2}=m_{\kappa}^{2}-\frac{q^{2}}{2} .
$$

Also, in Eq. (77) and in Figs. $1-3, Q^{2}=-q^{2}$ as usual.

For the pion electromagnetic form factor and pion radius, the expressions are given by (76) and (77) in the $m_{s}=m_{u}$ limit.

In Fig. 1 we plot the kaon electromagnetic form factor in the space-like region, evaluated in the present model compared with experimental data. This result shows a good agreement between the model and experiment. In Fig. 2 we focused the region near $Q^{2}=0$, that highlights the differences in the slopes of the $m_{u}=210 \mathrm{MeV}$ and $m_{u}=350 \mathrm{MeV}$ fittings, and explains why the difference in the kaon charge radius evaluated with these two sets of parameters is greater than it seems from Fig. 1. Fig. 3 presents the pion electromagnetic form factor.

In Fig. 4 we show the kaon weak decay constant for the up constituent mass in the range $210 \mathrm{MeV}<m_{u}<350 \mathrm{MeV}$. We can see that $f_{\kappa}$ decreases smoothly as $m_{u}$ increases.

In order to investigate if a different choice of $\lambda_{q}$ could provide a better fitting of the kaon weak decay constant in the $m_{u}=350 \mathrm{MeV}$ case, we also plot, in Fig. 5, the dependence of the kaon weak decay constant with $\lambda_{q}$. We verify that it is still not possible to fit the kaon weak decay constant with its experimental value for any value of $\lambda_{q}$. In fact, it is not possible to adjust 


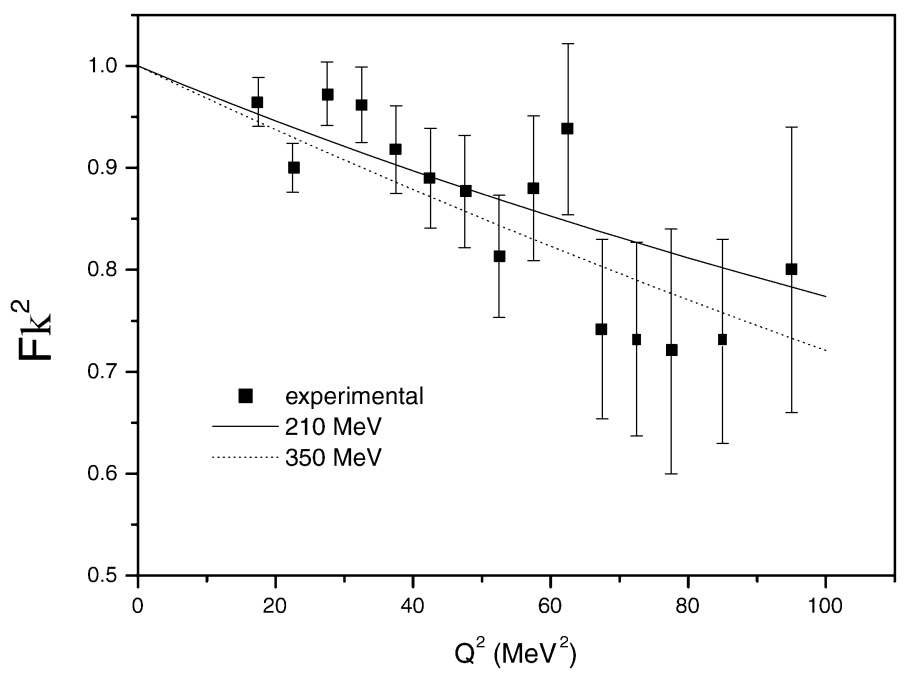

Fig. 1. Kaon electromagnetic form factor in the space-like region compared with experimental data [25]. Solid line corresponds to the set of parameters fitted with $m_{u}=350 \mathrm{MeV}$, dotted line corresponds to $m_{u}=210 \mathrm{MeV}$.

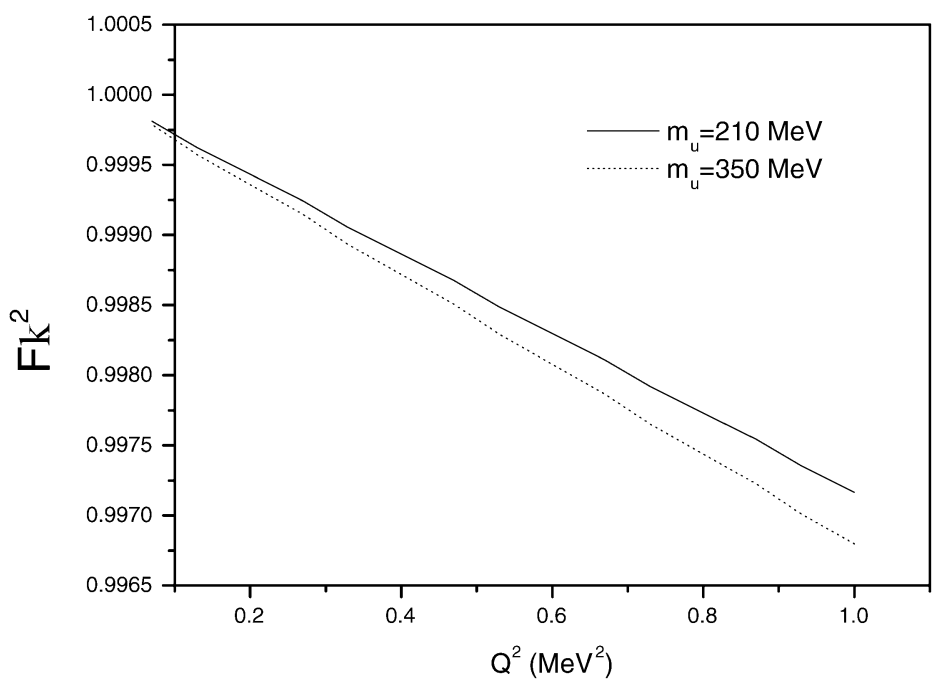

Fig. 2. Kaon electromagnetic form factor in the space-like region. Solid line corresponds to the set of parameters fitted with $m_{u}=350 \mathrm{MeV}$, dotted line corresponds to $m_{u}=210 \mathrm{MeV}$. The figure shows up the difference in the slope of the curves.

the parameters in order to reproduce $m_{\kappa}=494 \mathrm{MeV}$ for $\lambda_{q}<0.93$. For $0.93<\lambda_{q}<1.00$ we have $f_{\kappa}<71.6 \mathrm{MeV}$, and as $\lambda_{q}$ tends to infinity, the kaon weak decay constant tends to $f_{\kappa}=$ 94.6 MeV, and the constituent strange mass, which also depends on $\lambda_{q}$, runs from $m_{s}=644 \mathrm{MeV}$ to $m_{s}=350 \mathrm{MeV}$. 


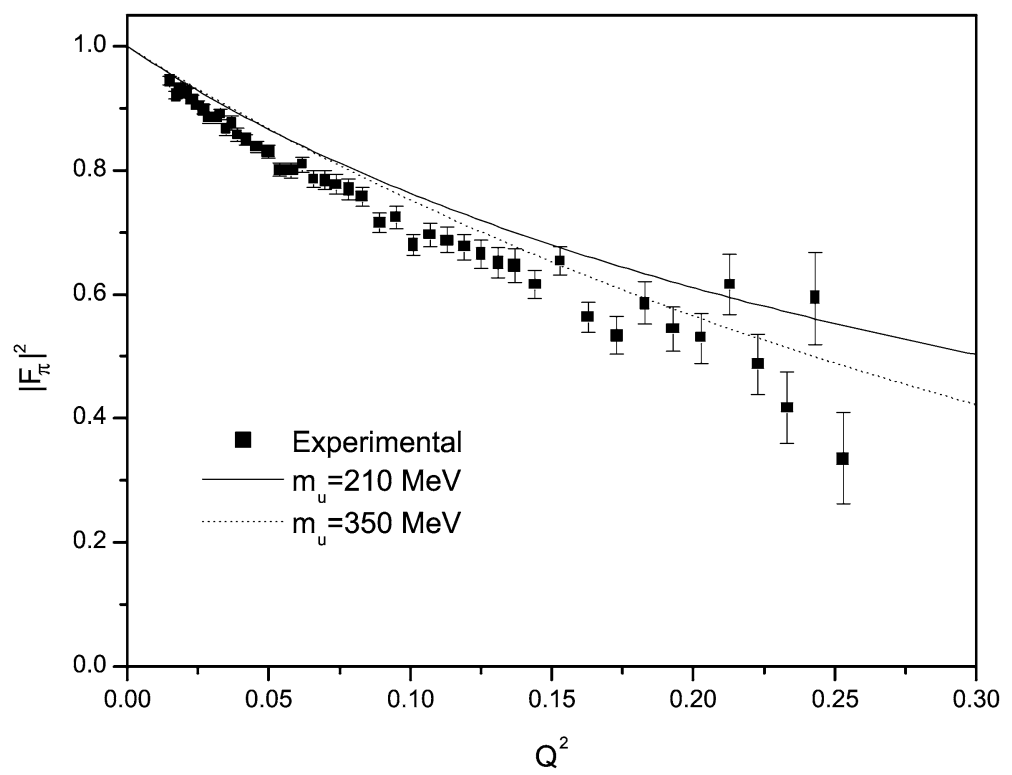

Fig. 3. Pion electromagnetic form factor in the space-like region compared with experimental data [26]. Solid line corresponds to the set of parameters fitted with $m_{u}=350 \mathrm{MeV}$, dotted line corresponds to $m_{u}=210 \mathrm{MeV}$.

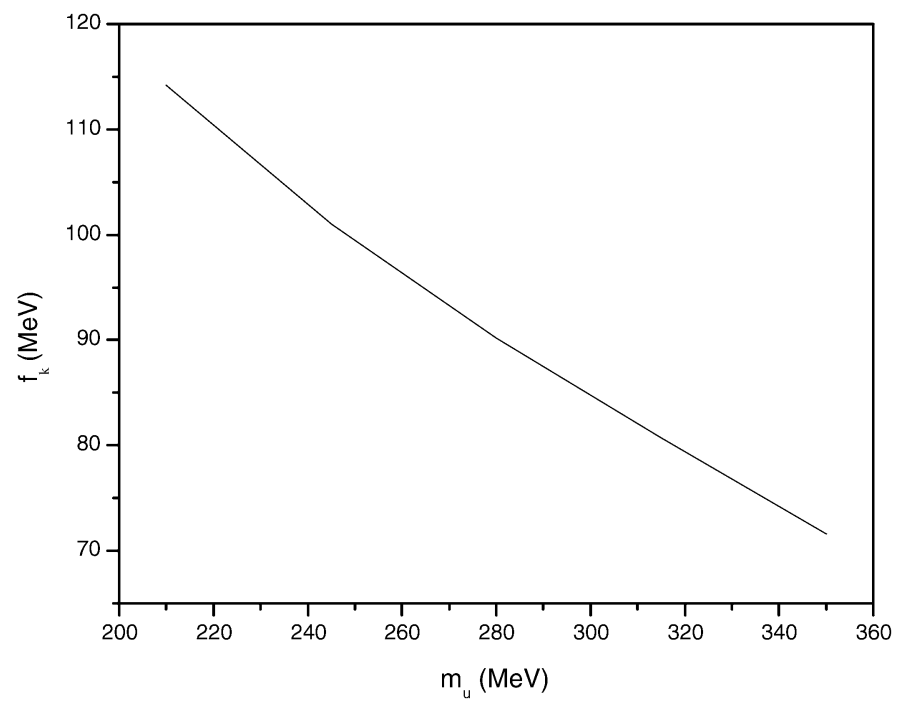

Fig. 4. Kaon weak decay constant for different quark up constituent masses.

\section{Conclusions}

We investigated the role of ambiguities associated with finite surface terms of the type of differences between logarithmic divergencies in the LSM with fermions. We found in the pseudoscalar-axialvector sector an arbitrary $c$-number which cannot be absorbed by renormalization nor fixed through the Goldberger-Treiman relation or PCAC. In this process, however, 


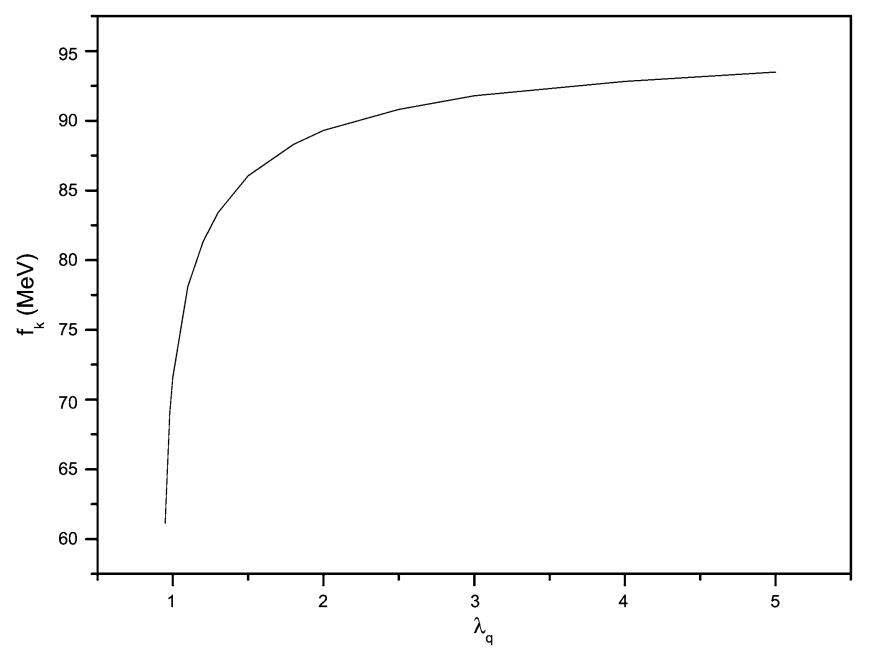

Fig. 5. Kaon weak decay constant as a function of $\lambda_{q}$ for $m_{u}=350 \mathrm{MeV}$.

necessary chiral and gauge invariant counterterms were introduced which link the pseudoscalaraxialvector sector with the scalar-vector one. The counterterms have a common coefficient which is fixed by the value assigned to the arbitrary $c$-number. If this number is chosen to be non-zero, it induces a symmetry breaking term in the scalar-vector sector. We must conclude therefore that the apriori arbitrary $c$-numbers are finally removed in order not to violate chiral symmetry in this sector. Further, we have obtained numerical results which show a good agreement between the model and experimental data for the pion and kaon electromagnetic form factors, even for higher values of the quark up constituent mass. The kaon weak decay constant for $m_{u}=350 \mathrm{MeV}$, nevertheless, cannot be adjusted close to its experimental value in the range of the other free model parameters.

\section{Acknowledgements}

This work has been supported by grants provided by Fundação para a Ciência e a Tecnologia, POCTI/35304/FIS/2000, POCTI/FNU/50336/2003, POCI/FP/63412/2005 and PRAXIS $\mathrm{XXI} / \mathrm{BCC} / 4301 / 94$. This research is part of the EU integrated infrastructure initiative HadronPhysics project under contract No. RII3-CT-2004-506078. A.A. Osipov also gratefully acknowledges the Fundação Calouste Gulbenkian for financial support and A.L. Mota acknowledges financial support from CNPq/Brazil and CAPES/Brazil.

\section{Appendix A}

Consider first the following example. The surface-term integral

$$
Y_{\mu \nu \alpha \beta}\left(m^{2}\right)=i \int \frac{d^{4} p}{(2 \pi)^{4}}\left[\frac{g_{\mu \nu \alpha \beta}}{\left(p^{2}-m^{2}\right)^{2}}-24 \frac{p_{\mu} p_{\nu} p_{\alpha} p_{\beta}}{\left(p^{2}-m^{2}\right)^{4}}\right]
$$

does not depend on $m^{2}$, i.e.

$$
\frac{d Y_{\mu \nu \alpha \beta}}{d m^{2}}=2 i \int \frac{d^{4} p}{(2 \pi)^{4}}\left[\frac{g_{\mu \nu \alpha \beta}}{\left(p^{2}-m^{2}\right)^{3}}-48 \frac{p_{\mu} p_{\nu} p_{\alpha} p_{\beta}}{\left(p^{2}-m^{2}\right)^{5}}\right]=0,
$$


where we use the standard notation

$$
g_{\mu \nu \alpha \beta}=g_{\mu \nu} g_{\alpha \beta}+g_{\mu \alpha} g_{\nu \beta}+g_{\mu \beta} g_{\nu \alpha} .
$$

Let us generalize Eq. (A.2). For that, note that in the space of $d=2 \omega$ dimensions one has

$$
g_{\mu_{1} \mu_{2} \ldots \mu_{2 n}} g^{\mu_{1} \mu_{2}} \cdots g^{\mu_{2 n-1} \mu_{2 n}}=2^{n} \frac{\Gamma(\omega+n)}{\Gamma(\omega)} .
$$

Therefore one obtains the identity

$$
\begin{aligned}
\int d^{2 \omega} p \frac{p_{\mu_{1}} p_{\mu_{2}} \cdots p_{\mu_{2 n}}}{\left(p^{2}-m^{2}\right)^{A}} & =\frac{i \pi^{\omega} \Gamma(A-\omega-n)}{\left(m^{2}\right)^{A-\omega-n} 2^{n} \Gamma(A)} g_{\mu_{1} \mu_{2} \ldots \mu_{2 n}} \\
& =\frac{\Gamma(A-n)}{2^{n} \Gamma(A)} \int d^{2 \omega} p \frac{g_{\mu_{1} \mu_{2} \ldots \mu_{2 n}}}{\left(p^{2}-m^{2}\right)^{A-n}} .
\end{aligned}
$$

Eq. (A.3) can be rewritten as

$$
\int d^{2 \omega} p\left[\frac{g_{\mu_{1} \mu_{2} \ldots \mu_{2 n}}}{\left(p^{2}-m^{2}\right)^{A-n}}-\frac{2^{n} \Gamma(A)}{\Gamma(A-n)} \frac{p_{\mu_{1}} p_{\mu_{2}} \cdots p_{\mu_{2 n}}}{\left(p^{2}-m^{2}\right)^{A}}\right]=0 .
$$

Integrating now with respect to $\mathrm{m}^{2}$ we obtain the most general form of all possible surface terms

$$
S_{\mu_{1} \mu_{2} \ldots \mu_{2 n}}^{(A-2)}=i \int \frac{d^{2 \omega} p}{(2 \pi)^{4}}\left[\frac{g_{\mu_{1} \mu_{2} \ldots \mu_{2 n}}}{\left(p^{2}-m^{2}\right)^{A-n-1}}-\frac{2^{n} \Gamma(A-1)}{\Gamma(A-n-1)} \frac{p_{\mu_{1}} p_{\mu_{2}} \cdots p_{\mu_{2 n}}}{\left(p^{2}-m^{2}\right)^{A-1}}\right] .
$$

\section{References}

[1] R. Jackiw, Int. J. Mod. Phys. B 14 (2000) 2001, hep-th/9903044.

[2] J.S. Bell, R. Jackiw, Nuovo Cimento A 60 (1969) 47.

[3] S.L. Adler, Phys. Rev. 177 (1969) 2426.

[4] A.P. Baêta Scarpelli, M. Sampaio, M.C. Nemes, B. Hiller, Phys. Rev. D 64 (2001) 046013, hep-th/0102108.

[5] O.A. Battistel, PhD thesis, Federal University of Minas Gerais, Brazil.

[6] A.P. Baêta Scarpelli, M. Sampaio, M.C. Nemes, Phys. Rev. D 63 (2001) 046004, hep-th/0010285;

M. Sampaio, A.P. Baêta Scarpelli, B. Hiller, A. Brizola, M.C. Nemes, S. Gobira, Phys. Rev. D 65 (2002) 125023, hep-th/0203261.

[7] S.R. Gobira, M.C. Nemes, Int. J. Theor. Phys. 42 (2003) 2765.

[8] D. Carneiro, A.P. Baêta Scarpelli, M. Sampaio, M.C. Nemes, JHEP 0312 (2003) 044, hep-th/0309188.

[9] L.A.M. Souza, M. Sampaio, M.C. Nemes, Phys. Lett. B 632 (2006) 717, hep-th/0510017.

[10] G. Bonneau, Nucl. Phys. B 593 (2001) 398, hep-th/0008210.

[11] A.M. Polyakov, Phys. Lett. B 59 (1975) 82;

A.M. Polyakov, Nucl. Phys. B 120 (1977) 429;

A.A. Belavin, A.M. Polyakov, A. Schwartz, Y. Tyupkin, Phys. Lett. B 59 (1975) 85;

G. 't Hooft, Phys. Rev. Lett. 37 (1976) 8;

G. 't Hooft, Phys. Rev. D 14 (1976) 3432;

C. Callan, R. Dashen, D.J. Gross, Phys. Lett. B 63 (1976) 334;

R. Jackiw, C. Rebbi, Phys. Rev. Lett. 37 (1976) 172;

S. Coleman, The uses of instantons, in: Erice Lectures, 1977;

G. 't Hooft, hep-th/9903189.

[12] M. Gell-Mann, M. Lévy, Nuovo Cimento 16 (1960) 705.

[13] S. Gasiorowicz, D.A. Geffen, Rev. Mod. Phys. 41 (1969) 531.

[14] Y. Nambu, G. Jona-Lasinio, Phys. Rev. 122 (1961) 345;

Y. Nambu, G. Jona-Lasinio, Phys. Rev. 124 (1961) 246;

V.G. Vaks, A.I. Larkin, Zh. Eksp. Teor. Fiz. 40 (1961) 282.

[15] J. Schwinger, Phys. Rev. 82 (1951) 664.

[16] B. DeWitt, Dynamical Theory of Groups and Fields, Gordon \& Breach, New York, 1965. 
[17] R.D. Ball, Phys. Rep. 182 (1989) 1, and references therein.

[18] A.A. Osipov, H. Hansen, B. Hiller, Nucl. Phys. A 745 (2004) 81, hep-ph/0406112.

[19] T. Eguchi, Phys. Rev. D 14 (1975) 2755.

[20] H. Reinhardt, R. Alkofer, Phys. Lett. B 207 (1988) 482.

[21] E.N. Nikolov, W. Broniowski, C.V. Christov, G. Ripka, K. Goeke, Nucl. Phys. A 608 (1966) 411, hep-ph/9602274.

[22] V. Bernard, A.H. Blin, B. Hiller, Y.P. Ivanov, A.A. Osipov, U.-G. Meißner, Ann. Phys. 249 (1996) 499;

A.A. Osipov, B. Hiller, A.H. Blin, Phys. Lett. B 475 (2000) 324, hep-ph/9912404;

A.A. Osipov, B. Hiller, Phys. Rev. D 62 (2000) 114013, hep-ph/0007102;

A.A. Osipov, B. Hiller, Phys. Rev. D 63 (2001) 094009, hep-ph/0012294;

A.A. Osipov, M. Sampaio, B. Hiller, Nucl. Phys. A 703 (2001) 378, hep-ph/0110285.

[23] E.W. Dias, B. Hiller, A.L. Mota, M.C. Nemes, M. Sampaio, A.A. Osipov, Mod. Phys. Lett. A 21 (2006) 339, hep-ph/0503245.

[24] A.L. Mota, M.C. Nemes, B. Hiller, H. Walliser, Nucl. Phys. A 652 (1999) 73, hep-th/9901455.

[25] S.R. Amendolia, et al., Phys. Lett. B 178 (1986) 435.

[26] S.R. Amendolia, et al., Phys. Lett. B 138 (1984) 454. 